\title{
A Progressive Cut Refinement Scheme for Revision Total Hip Replacement Surgery Using C-arm Fluoroscopy
}

\author{
Jianhua Yao ${ }^{1}$, Russell H. Taylor ${ }^{1}$, Randal P. Goldberg ${ }^{1}$, Rajesh Kumar ${ }^{1}$, \\ Andrew Bzostek ${ }^{1}$, Robert Van Vorhis ${ }^{2}$, Peter Kazanzides ${ }^{2}$, \\ Andre Gueziec $^{3}$, and J. Funda ${ }^{3}$ \\ ${ }^{1}$ Computer Science Department, The Johns Hopkins University, Baltimore, MD, USA \\ ${ }^{2}$ Integrated Surgical Systems, Davis, CA, USA \\ ${ }^{3}$ IBM T.J. Watson Research Center, Yorktown Heights, NY, USA
}

\begin{abstract}
We describe a new method to cut a precise, high quality cavity in Revision Total Hip Replacement surgery (RTHR) using a set of intra-operative $\mathrm{C}$-arm fluoroscopic images. With respect to previous approaches, our method provides the following new features: (1) a novel checkerboard plate was designed to correct the geometric distortion within fluoroscopic images. Unlike previous distortion correction devices, the plate doesn't completely obscure any part of the image, and the distortion correction algorithm works well even when there are some overlaid objects in field of view; (2) a novel corkscrew fiducial object attached to the robot end-effector was designed, and a $6 \mathrm{D}$ pose estimation algorithm based on the $2 \mathrm{D}$ projection of the corkscrew is developed and used in robot-imager registration and imager co-registration; (3) we propose a progressive cut refinement scheme and an iterative cut location algorithm which utilizes image subtraction and 2D anatomy contour matching techniques. Several cutting experiments and some simulated experiments have been conducted to assess our techniques. The results indicate that our scheme is a promising method for RTHR application.
\end{abstract}

\section{Introduction and Previous Works}

Our research is part of a joint project with Integrated Surgical System (ISS), at Davis, CA., to develop a computer integrated system to assist surgeons in revision total hip replacement surgery (RTHR). RTHR is performed after a patient had PTHR (Primary Total Hip Replacement Surgery) and the implant fails for some reason. RTHR is a much more difficult operation, because less bone tissue remains and a precise, high quality canal is required [1].

In PTHR and RTHR, a surgical robot is needed to mill a precise cavity for the femoral implant. The robot trajectory is planned preoperatively based on a CT scan of the femur and the CAD model of the implant. Then the robot trajectory is executed after the intra-operative registration between the robot and the femur [1,2]. Accurate robot-to-patient and robot-to-image registrations are essential to RTHR surgery. Reg- 
istration techniques have included: implanted fiducials as in ROBODOC $^{\circledR}$ [3], 3D-3D anatomy based registration [4], and 2D-3D anatomy based registration [4-6]. Among these, 2D-3D anatomy based registration is the most favorable one because it is less invasive, but it also presents significant technical challenges.

Our developments of a computer integrated RTHR system started about four years ago. Taylor, Joskowicz et al. $[1,2]$ proposed the initial system. Some of their ideas have been investigated in the current research. Gueziec et al. [5, 7] explored the feasibility of anatomy based registration using fluoroscopy. The goal of our research is to direct the robot to cut a precise cavity using intra-operative portable $\mathrm{C}$-arm fluoroscopy as guidance. We propose a series of new methods and system to solve this problem. Section 2 addresses the overview of the system first. Then a new method for fluoroscopic image spatial distortion correction is described in section 3 . In section 4 we present a novel pose estimation device that can be attached to the robot endeffector and provide a method to compute the transformation between robot and imager geometry and as well as perform co-registration between different imager geometry. In section 5, the experimental protocol is introduced. A progressive cut refinement scheme is elaborated in section 6. Finally we discuss several experiments we have done so far and our future plan.

\section{System Overview}

Our current work is applicable for execution of pre-surgical plans based either on preoperative CT images or on multiple intra-operative 2D X-ray images. The flow chart in Figure 1(left) illustrates the former option. First the cut cavity is planned based on the preoperative CT volume. During preoperative setup, a corkscrew-shaped fiducial object is mounted on the robot's cutter and a calibration procedure is performed to determine the corkscrew-to-robot transformation (section 4). In the intraoperative stage, the femur is placed in a fixation device that holds it in a fixed but unknown position relative to the robot. Multiple $\mathrm{C}$-arm images are grabbed. The registrations among robot, patient and imager are conducted using our pose estimation algorithm and the anatomy or fiducial based registration method (section 4, 5). Then a small progressive cut is executed by the guidance of the images. After the cut, another set of images is taken in similar $\mathrm{C}$-arm poses as those used for initial registration. The real cut cavity is detected using a digital subtraction technique and is compared to the expected cavity (section 6). Then the registration between the robot and patient is updated based on the discrepancy between the real cavity and the expected cavity. The procedure is iterated until we get the final cut.

A typical flow for intraoperative X-ray based plans is shown in Figure 1(right). Such a case can arise in RTHR if CT reconstruction artifacts are so severe that CTbased planning is impractical or if unexpected circumstances during surgery make replanning necessary. In this case, the femur is placed into a fixation device that holds it stationary with respect to the robot and multiple C-arm X-ray images are taken as before. A cut cavity shape is determined and its desired pose is determined interactively using an "image spreadsheet" $[1,2]$ in which the projected contours of the cavity are superimposed on the X-ray images. Iterative cutting proceeds as before. 
We have prototyped our method and experimentally demonstrated it on dental acrylic phantoms and simulated images. Figure 2 is a typical experimental setup. The de-warping plate is placed over the $\mathrm{C}$-arm detector. The corkscrew for pose estimation is attached to a ROBODOC ${ }^{\circledR}$ cutter. A dental acrylic phantom held by a box holder is used for these cutting experiments to evaluate our method.

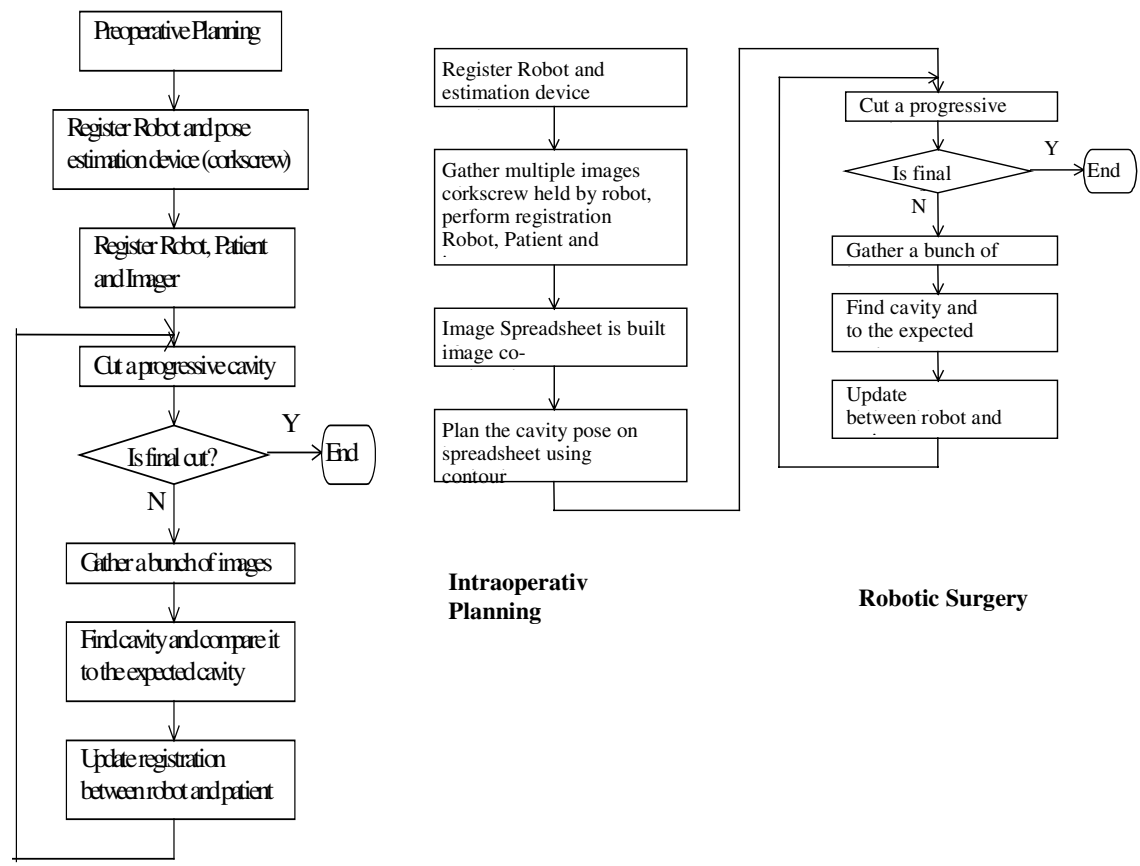

Figure 1. (left) Procedure flow for CT-based plans;

(right) Procedure flow for multiple x-ray based plans.

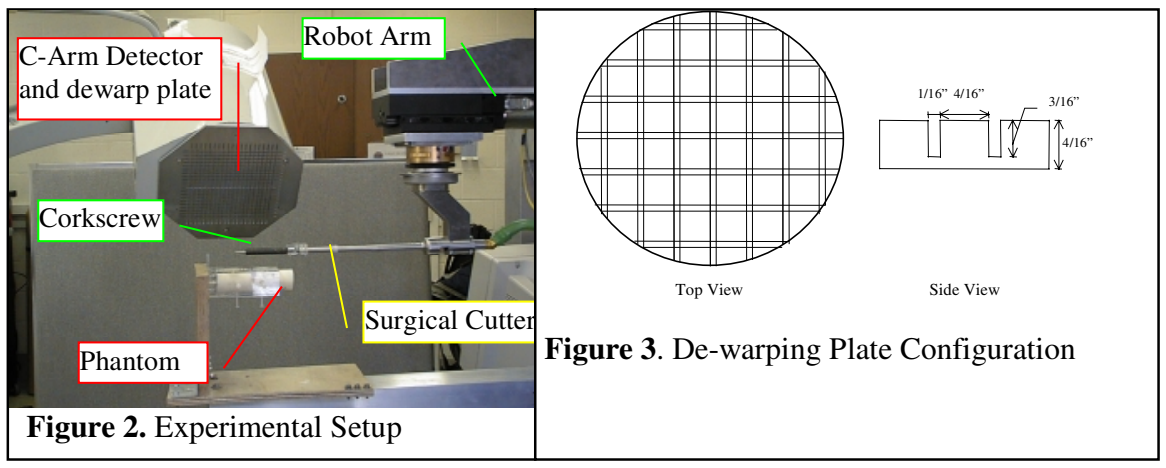




\section{Intrinsic Image Calibration}

Intrinsic imaging parameters correspond to image warping, focal length, pixel scaling, and image center, and can be computed by analyzing an image of a calibration object. Schreiner's method [8] was used to compute the C-arm focal length, the pixel scaling and the image center. We assume that these parameters do not change under different $\mathrm{C}$-arm poses. Using fluoroscopic X-ray imaging for quantitative measurement requires precise calibration of the imager to remove the spatial distortions caused by the intensifier. Boone [9] provided the theory and analysis of the nature of the distortion and proposed some techniques to correct it. Schreiner [8] implemented

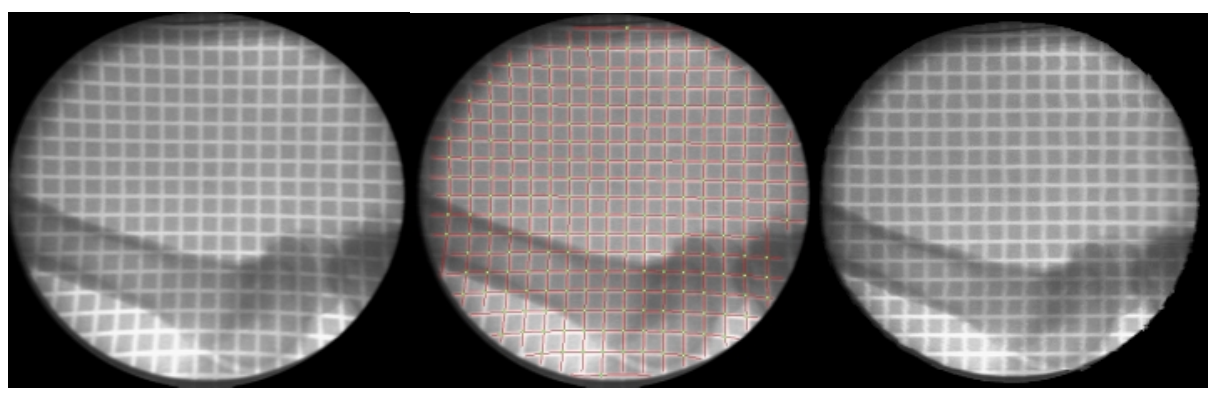

Figure 4: Image de-warping Results. (Left) Fluoroscopic x-ray image of a smoked ham. The white lines correspond to grooves cut into an aluminum de-warping calibration plate. (Middle) The same image after the grooves have been located. (Right) De-warped image.

Boone's method by placing a grid of radiopaque beads over the $\mathrm{C}$-arm detector.

In our distortion correction approach, a $1 / 4$ inch thick semi-radiolucent aluminum plate is placed over the detector of the fluoroscopic C-arm (Figure 2). Horizontal and vertical grooves with 3/16 inches deep and 1/16 inch wide are machined in the plate in a square pattern on 1/4 inch intervals (Figure 3 ). These grooves show up as pale lines on the x-ray images, and provide enough contrast to be found in the log image by delicate image processing methods. Other objects are still clearly visible with the checkered pattern as background. It has some advantages for cases such as ours, where the $\mathrm{C}$-arm may be placed in multiple unpredictable poses during the procedure, thus making a separate preoperative distortion correction calibration impractical. In particular, the checkerboard geometry does not completely obscure any part of the image, allowing it to remain in place during the procedure although there is some sacrifice of contrast.

We have investigated various ways to use this checkerboard plate to compensate for fluoroscopic image distortion. Methods examined include Piecewise Polynomial Mapping Algorithm, Thin Plate Spline Morphometric Algorithm [10], and Two-Pass Scanline Algorithm [11]. Our current preferred choice is a variant of Two-Pass Scanline Algorithm. Sample images can be found in Figure 4.

The accuracy of the distortion correction process was verified by following experiments: First one image with the checkerboard plate was taken and the spatial dis- 
tortion of the image was corrected, and the lookup table was saved. Then a bead pattern was attached over the plate, and another image was taken. The bead pattern image was then de-warped using the saved lookup table. The beads were detected in the image and distances between pairs of beads computed. Comparison of the computed distance and the actual distance between beads in the bead pattern gives an assessment of the accuracy of the de-warping algorithm. The mean error was $0.12 \mathrm{~mm}$ on the central area and $0.25 \mathrm{~mm}$ on the marginal area while the beads were $20 \mathrm{~mm}$ apart. (Pixel size is approximately $0.32 \mathrm{~mm}$ ). This shows that our distortion correction methods provide reasonably accurate results.

\section{C-arm Pose Estimation and Extrinsic Calibration}

For extrinsic calibration, the task is to compute the transformation between the Carm coordinate system and other intra-operative coordinate systems such as the patient's anatomy, the robot, and the surgical tool.

Our current research provides image-based methods for the co-registration of the portable $\mathrm{C}$-arm without extrinsic tracking device. Our strategy relies on identifying features within a single X-ray image of a known 3D arrangement of fiducials and computing the appropriate transformation. These considerations have led us to explore a calibration fiducial geometry consisting of a 5/4-turn "corkscrew" spiral and four circular rings surrounding a central shaft, as shown in Figure 5. The initial embodiment is designed to be held in the JHU/IBM LARS ${ }^{\mathrm{TM}}$ robot and the ROBODOC ${ }^{\circledR}$ orthopedic cutter. It is fabricated from autoclavable polyamide with a steel central shaft and copper wire filled grooves machined into the outside of the polyamide rod.
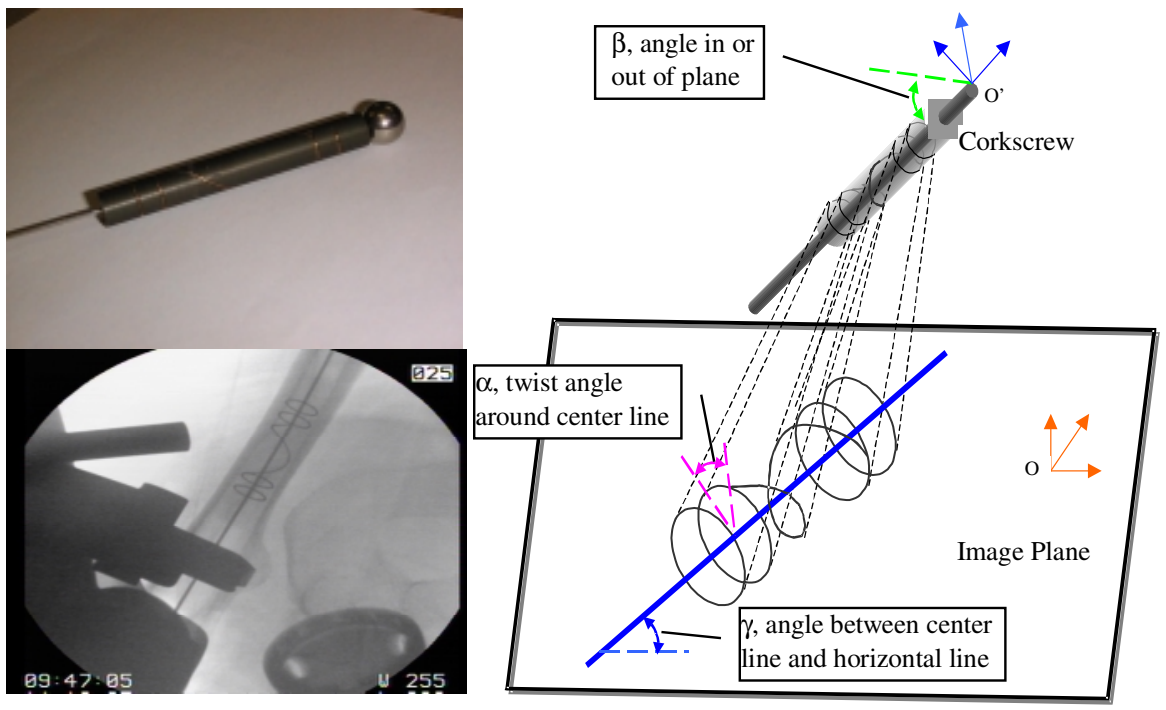

Figure 5. Corkscrew fiducial object. (left top) Corkscrew fiducial object. (left bottom) Introaoperative $\mathrm{x}$-ray image of corkscrew object. (right) Projection of corkscrew 
The fiducial geometry of the corkscrew has the property that its $6 \mathrm{D}$ pose can be computed from a single projected 2D image. Calculation of these parameters is accomplished by applying delicate image segmentation algorithms and a series of Hough transforms to the subject image, calculating a subset of the parameters using different parts of the fiducial geometry as following (Figure 5): The centerline of the shaft provides one rotation and one translation parameter, both in the plane of the image, restricting the centerline of the fiducial to lie on a plane in 3D space. The ellipses projected by the four rings give the other two translations as well as a rotation into or out of that plane. The first translation is determined by the position of the pattern of ellipses along the centerline. And the distance between the ellipses determines the fiducial's distance from the image plane (one translation in 3D). The phase angle of the projection of the helix directly determines the twist rotational angle. The shape of the projected helix also determines whether the corkscrew rotates into or out of the image plane, i.e. the sign of angle $\beta$. After finding and computing the geometry parameters of the corkscrew projection on the $2 \mathrm{D}$ image, the transformation between the image coordinate system $(\mathrm{O})$ and the corkscrew coordinate system $\left(\mathrm{O}^{\prime}\right)$ can be constructed. The algorithm is pretty robust due to the use of the Hough transform and the geometric constraints between different parts. The corkscrew can be used for pose estimation of $\mathrm{C}$-arm geometry and co-registration between two $\mathrm{C}$-arm poses. By attaching this corkscrew to the robot's end effector and conducting a pre-operative corkscrew-robot registration, the registration between the robot and the imager can be computed.

We conducted the following experiments to assess the accuracy of our method. The corkscrew was mounted on the LARS ${ }^{\mathrm{TM}}$ robot's end-effector. One image of the corkscrew was taken first. Keeping the $\mathrm{C}$-arm pose unaltered, the robot joint was then translated or rotated. Then another image was taken after the movement. The corkscrew movement (i.e. the movement of the robot end-effector) between the two images was computed based on the pose estimation algorithm. Then the computed movement was compared to the real movement of the robot. Table 1 and Table 2 show the results. The results indicate that the rotation accuracy is around $1^{\circ}-2^{\circ}$. The error of the roll angle is large because the secondary radius of the ring can not be computed accurately especially when the angle is small. The results also show that the average translation error is under $0.5 \mathrm{~mm}$ along the direction parallel to the image plane (Axis $\mathrm{x}, \mathrm{y}$ ), and can be larger than $1.0 \mathrm{~mm}$ along the focal length direction (Axis $\mathrm{z}$ ).

\begin{tabular}{|c|c|c|c|c|c|c|c|c|c|}
\hline \multirow[t]{2}{*}{ Trial } & \multicolumn{3}{|c|}{ Robot Rotation Angle $\left(^{\circ}\right)$} & \multicolumn{3}{|c|}{ Computed Rotation Angle $\left(^{\circ}\right)$} & \multicolumn{3}{|c|}{ Error $\left(^{\circ}\right)$} \\
\hline & Twist & Roll & Pitch & Twist & Roll & Pitch & Twist & Roll & Pitch \\
\hline 1 & 30 & 15 & 15 & 30.28 & 17.26 & 14.82 & 0.28 & 2.26 & 0.18 \\
\hline 2 & 60 & 30 & 30 & 59.53 & 31.76 & 29.34 & 0.47 & 1.76 & 0.66 \\
\hline 3 & 90 & 45 & 45 & 88.95 & 44.17 & 47.07 & 1.05 & 0.83 & 2.07 \\
\hline \multicolumn{7}{|c|}{ Average Error } & $0.60^{\circ}$ & $1.62^{\circ}$ & $0.97^{\circ}$ \\
\hline \multicolumn{7}{|c|}{ Standard Deviation } & $0.33^{\circ}$ & $0.59^{\circ}$ & $0.80^{\circ}$ \\
\hline
\end{tabular}

Table 1. Rotation Error Assessment in Corkscrew Pose Estimation Method 


\begin{tabular}{|c|c|c|c|c|c|c|c|c|c|}
\hline Trial & \multicolumn{3}{|c|}{ Robot Translate (mm) } & \multicolumn{3}{|c|}{ Computed Translate $(\mathrm{mm})$} & \multicolumn{3}{|c|}{ Error $(\mathrm{mm})$} \\
\hline & $\mathrm{X}$ & $\mathrm{Y}$ & $\mathrm{Z}$ & $\mathrm{X}$ & $\mathrm{Y}$ & $\mathrm{Z}$ & $\mathrm{X}$ & $\mathrm{Y}$ & $\mathrm{Z}$ \\
\hline 1 & 5 & 5 & 5 & 5.04 & 5.13 & 5.61 & 0.04 & 0.13 & 0.61 \\
\hline 2 & 10 & 10 & 10 & 9.73 & 10.39 & 11.25 & 0.27 & 0.39 & 1.25 \\
\hline 3 & 15 & 15 & 15 & 14.2 & 14.35 & 13.62 & 0.8 & 0.65 & 1.38 \\
\hline \multicolumn{7}{|c|}{ Average Error } & 0.37 & 0.39 & 1.08 \\
\hline \multicolumn{7}{|c|}{ Deviation } & 0.32 & 0.21 & 0.34 \\
\hline
\end{tabular}

Table 2. Translation Error Assessment in Corkscrew Pose Estimation Method

\section{Cutting Experiment}

We have conducted some cutting experiments using the orthopedic cutter and dental acrylic phantoms. Extensive tests were carried out on plastic bones and foam test blocks in order to verify basic system accuracy and to gain confidence in overall system behavior. The following is a general procedure for a cutting experiment.

Step 1: The corkscrew is attached to the cutter mounted on the end-effector. A separate procedure is performed to calibrate the robot and the corkscrew, i.e. $F_{\text {robot-corkscrew }}$. Step 2: Several images of the corkscrew and the phantom are taken. Then the registration, $F_{\text {corkscrew-imager }}$, between the corkscrew and the imager is computed using the corkscrew pose estimation algorithm in Section 3 . The registration $F_{\text {femur-imager }}$ between the femur and the imager is obtained using the femur anatomy or fiducial beads (at present fiducial beads are used). Finally the registration between femur and robot is written as $F_{\text {robot-femur }}=F_{\text {robot-corkscrew }} \cdot F_{\text {corkscrew-imager }} \cdot F_{\text {imager-femur }}$

Step 3: The cutter is moved to the starting position, and a predefined shape is cut.

The cavity position was estimated by measuring wall thickness using calipers to get the assessment of the errors. With the angular separation of about 50 degree between two $\mathrm{C}$-arm views, we observe a cavity placement error of about $0.5 \mathrm{~mm}$ in one direction in the cutting plane but as large as $1.5 \mathrm{~mm}$ in the other direction. A number of factors may count for this result: the limited angular separation between views, the particular method used to cut the test cavity, the calibration error in setup. In any case, these results led us to explore a progressive cutting scheme described in Section 6.

\section{Iterative Cut Cavity Location Algorithm}

We are investigating a progressive cutting scheme to improve the placement accuracy: First a small cavity is cut. Then an iterative cut cavity location algorithm is applied to compute the discrepancy between the real cut pocket and the cut model, then the registration between the robot and femur anatomy is adjusted accordingly. On the next cut, a larger pocket is cut and the process is repeated until the final desired shape is cut. The idea of progressive cutting is straightforward. The problem is how to measure the error after each cut using the fluoroscopic C-arm. We developed an iterative cut cavity location algorithm to solve this problem.

The idea of the iterative cut cavity location algorithm is outlined: The cut cavity model and its transformation relative to the femur is planned in the pre-operative stage. After each cut, several images of the cut cavity and corkscrew from different 
view angles are taken. The $\mathrm{C}$-arm geometry of each image is constructed using the corkscrew pose estimation algorithm. On each image, an image subtraction technique (section 6.3) is employed to generate the 2D contour of the cut cavity, denoted as $C_{c}$. The projective apparent contour $C_{m}$ (section 6.1) of the cut cavity model is built. A 2D contour registration algorithm (section 6.2) is applied to get the $2 \mathrm{D}$ transformation between $C_{c}$ and $C_{m}$. Then the $2 \mathrm{D}$ transformation between contours is back-projected to a $3 \mathrm{D}$ transformation. Finally the transformation between the real cut cavity and the cut model is updated (Section 6.4). The above procedure is iterated on all images. Then the transformation is used to update the registration between the robot and the femur.

\subsection{Projective Apparent Contour of 3D Model}

An algorithm to generate the $2 \mathrm{D}$ projective apparent contour of a 3D surface model was developed. A 3D surface model is a list of 3D triangular facets. Given the view geometry, the projective apparent contour is the projection of a set of edges on the surface, such that the facet on one side of the edge is visible while the facet on the other side is invisible.

\subsection{D Contour Registration Algorithm}

There are two stages in the computation of the 2D transformation between two contours. During the first stage the initial 2D transformation is computed using first and second order moments of the contour. Second stage is an Iterative Closet Point algorithm. It involves using Least-Square-Error method to recursively update the transformation. It usually takes two or three iterations to converge in the second stage.

\subsection{Digital Subtraction}

On the image of the cut cavity, the edge of the cavity can be very blurred, it is impractical and inaccurate to rely on an edge detector or image gradient to detect the contour of the cavity. We turned to digital subtraction technique for solutions [12]. One image is taken before the cutting and the C-arm pose is marked. After the cut is done, the $\mathrm{C}$-arm is moved back to the marked position and another image is taken. The digital subtraction of these two images can provide meaningful information. Under ideal conditions, this subtraction image can detect subtle changes. If the two Carm poses are different, structured noise exists. Fortunately in our case the C-arm pose is marked, so the difference between two imager poses should be small. Furthermore the outer contour of the femur hardly changes before and after the cut, the 2D contour transformation algorithm in section 6.2 can be employed on them to compute the 2D transformation between two images. Then one image is rotated, translated and scaled to make the two images have maximal mutual information. After that the two images can be subtracted without too much structured noise. We tested this idea on some simulated images generated from a CT set. (Figure 6). 


\subsection{Computational Analysis}

The objective of this algorithm is to compute $\Delta F$, i.e. the transformation between the real cavity and the cavity model.

The cavity model frame relative to the $\mathrm{C}$-arm coordinate system can be written as $F_{v-m}=F_{v}^{-1} F_{m}$, where $F_{v}$ is the C-arm coordinate frame and $F_{m}$ is the cut cavity model coordinate frame. Based on $F_{v-m}$, the model projective contour $C_{m}$ is generated, then the $2 \mathrm{D}$ contour transformation algorithm is employed to get the $2 \mathrm{D}$ translation $(\Delta x, \Delta y), 2 \mathrm{D}$ rotation angle $\Delta \theta$ and rotation center $\left(x_{0}, y_{0}\right)$.

Distance from the cavity model to the $\mathrm{C}$-arm source along the focal length direction can be written as $d=\left(F_{m} \cdot P-F_{v} \cdot P\right) \circ F_{v} \cdot R \cdot R z$, where $F_{v} \cdot R \cdot R z$ is the viewing direction, and $\circ$ is the dot product between two vectors.

The 3D transformation between the real cavity and the cavity model $\Delta F_{c}=T_{2}^{-1} R_{1} T_{2} T_{1}$, and $T_{1}=\operatorname{Translate}\left(\Delta x \cdot s_{x} \cdot d / f, \Delta y \cdot s_{y} \cdot d / f, 0\right), R_{1}=$ Rotation $(\Delta \theta, Z-$ Axis $)$ ,$T_{2}=\operatorname{Translate}\left(x_{0} \cdot s_{x} \cdot d / f, y_{0} \cdot s_{y} \cdot d / f, 0\right)$, and $f$ is the focal length of the C-arm and $\left(s_{x}, s_{y}\right)$ is the pixel size of the $2 \mathrm{D}$ image.

So the cumulative transformation $\Delta F_{w}$ can be denoted as $\Delta F_{w} \cdot P=F_{v} \cdot R \cdot \Delta F_{c} \cdot P$ and $\Delta F_{w} \cdot R=F_{v} \cdot R \cdot \Delta F_{c} \cdot R$. Then the Cavity Model frame and the cumulative transformation can be updated by: $F_{m}=\Delta F_{w} \cdot F_{m}$ and $\Delta F=\Delta F_{w} \cdot \Delta F$

The above computing procedure is iterated on all images. Finally the transformation between the real cavity and the cavity model is got.

\subsection{Results}

We have tested our ideas on some simulated images. An algorithm was developed to generate the simulated fluoroscopic image from a CT data volume based on the attenuation rule. The simulated image is very realistic except that there is no spatial distortion. The process of cutting a pre-defined cavity from the CT volume can also be simulated by subtracting the cavity volume from the CT volume. Figure 6 is a set of simulation images generated from a CT set of a patient femur.

Table 3 provides some numerical assessment of our method. During this experiment, a cut cavity (in the shape of an implant) is first preplanned relative to the femur. Then the cut cavity is perturbed with various transformations, and a simulated cutting of the perturbed cavity is executed on the femur. Then the Iterative Cavity Location algorithm is employed to recover the perturbation transformation. Figure 6 illustrates the images simulated in trial 4 of table 3 . 


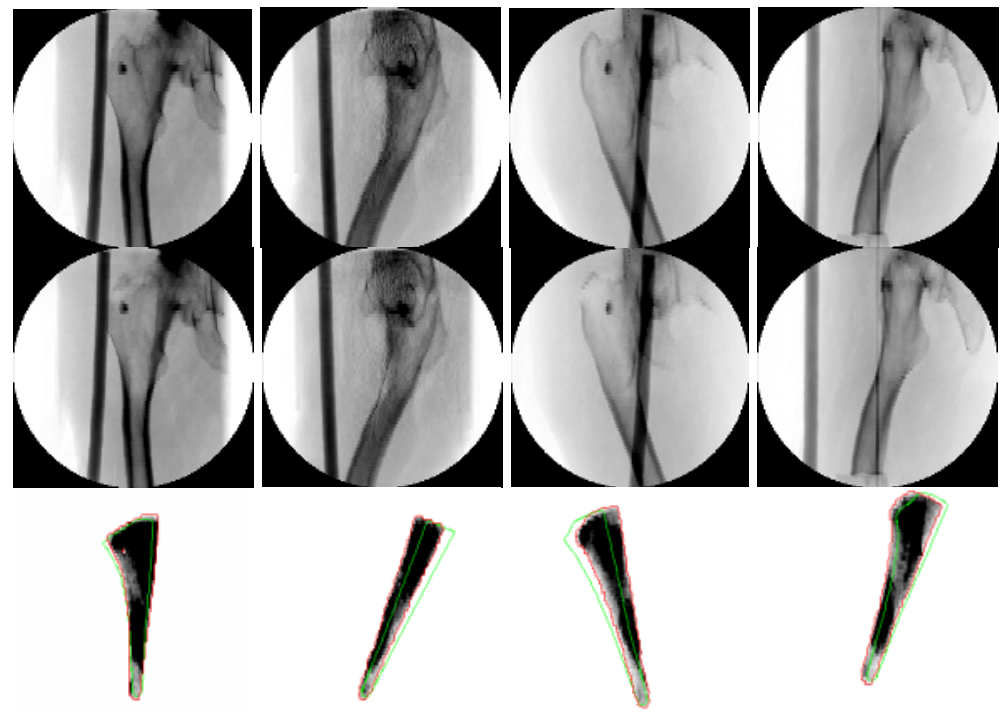

Figure 6 Images Used on Iterative Cavity Location Algorithm. Row 1 are images before the cutting; row 2 are those after cutting; and row 3 are their subtraction after removing the noises and enhancing the contrast, and superimposed by the contours of real cavity (red

\begin{tabular}{|c|c|c|c|c|c|c|c|c|c|c|c|c|c|c|}
\hline & \multicolumn{6}{|c|}{ Expected Transformation } & \multicolumn{6}{|c|}{ Computed Transformation } & \multirow{2}{*}{$\begin{array}{l}\text { Trans } \\
\text { Error } \\
(\mathrm{mm})\end{array}$} & \multirow{2}{*}{$\begin{array}{c}\text { Rota- } \\
\text { tion } \\
\text { Error } \\
\left({ }^{\circ}\right) \\
\end{array}$} \\
\hline $\begin{array}{c}\text { Tria } \\
1\end{array}$ & $\mathrm{X}$ & $\mathrm{Y}$ & $\mathrm{Z}$ & Roll & Pitch & Yaw & $\bar{X}$ & $\mathrm{Y}$ & $\mathrm{Z}$ & Roll & Pitch & Yaw & & \\
\hline 1 & 2.00 & 1.00 & 0.00 & 0.00 & 0.00 & 0.00 & 1.93 & 1.05 & 0.01 & 0.00 & 0.00 & 0.00 & \begin{tabular}{|l|}
0.09 \\
\end{tabular} & 0.00 \\
\hline 2 & -2.00 & 1.50 & 1.00 & 0.00 & 0.00 & 0.00 & \begin{tabular}{|l|}
-2.09 \\
\end{tabular} & 1.42 & 0.94 & 0.03 & 0.00 & $\mid-0.01$ & 0.13 & 0.03 \\
\hline 3 & 0.00 & 0.00 & 0.00 & -3.00 & 3.00 & 3.00 & 0.11 & -0.05 & 0.07 & \begin{tabular}{|l}
-2.18 \\
\end{tabular} & 3.49 & \begin{tabular}{|l|}
1.67 \\
\end{tabular} & 0.14 & 1.64 \\
\hline & 1.00 & -2.00 & -3.00 & 3.00 & 2.00 & 2.00 & 0.56 & -2.21 & -3.30 & 2.47 & 2.37 & 0.43 & 0.57 & 1.70 \\
\hline \multicolumn{13}{|c|}{ Average } & 0.23 & 0.84 \\
\hline \multicolumn{13}{|c|}{ Std Dev } & 0.23 & 0.96 \\
\hline
\end{tabular}

Table 3 Results of Recursive Cavity Location Algorithm

\section{Discussions and Future Plans}

The system and method described in this paper demonstrates the feasibility of cutting a precise pocket using the $\mathrm{C}$-arm fluoroscopy. We have been able to demonstrate an-order-of magnitude improvement in the precision by the progressive refinement scheme in revision total hip replacement (RTHR) surgery. Based on the preliminary results, our research shows that a fluoroscopy based registration method and progressive cutting scheme is a promising alternative for RTHR as well as other orthopedic surgery procedures.

A number of areas must be further investigated, including the anatomy based registration methods. We plan to further assess the accuracy of the corkscrew pose estimation algorithm under different circumstance. We also plan to integrate an independent validation system to assess the accuracy of our system. 


\section{Acknowledgements}

This work was partially funded by NIST Advanced Technology Program Cooperative Agreement No. 94-01-0228. It benefited from NSF equipment grants CDA-9529509 and EIA9703080 and from an equipment grant from Intel. We thank our colleagues from IBM and ISS for their collaboration in all phases of this research. Leo Joskowicz, Alan Kalvin, Bill Williamson, and Brent Mittelstadt perhaps deserve special thanks, as does Rod Turner, MD from New England Baptist Hospital.

\section{Reference:}

[1] R. H. Taylor, L. Joskowicz, et al, "Computer-Integrated Revision Total Hip Replacement Surgery: Concept and Preliminary Results,” Medical Image Analysis, vol. 3, pp. 1-18, 1999.

[2] L. Joskowicz, R. H. Taylor, et al, "Computer Integrated Revision Total Hip Replacement Surgery: Preliminary Report”, pp. 193-202, presented at MRCAS, 1995.

[3] R. H. Taylor, B. D. Miittelstadt, et al, "An Image-Directed Robotics System For Precise Orthopaedic Surgery," in Computer-Integrated Surgery, Technology and Clinical Applications, R. H. Taylor, Ed., 1995, pp. 379-396.

[4] S. Lavallee, et al, "Anatomy-Based Registration of Three Dimensional Medical Images, Range Images, X-Ray Projections, and Three Dimensional Models Using Octree-Splines," in Computer-Integrated Surgery, R. H. Taylor, et al, Eds., 1995, pp. 115-144.

[5] A. Gueziec, P. Kazanzides, et al, "Anatomy Based Registration of CT-scan and Intraoperative X-ray Images for Guiding a Surgical Robot," IEEE Transactions on Medical Imaging, special issue on IMAGE GUIDANCE OF THERAPY, 1998.

[6] A. Hamadeh, P. Sautot, et al, "Towards automatic registration between CT and X-ray images: cooperation between 3D/2D registration and 2D edge detection," presented at Second Annual International Symposium on MRCAS, Baltimore, MD USA, 1995.

[7] A. P. Gueziec and J. Funda, "Evaluating the Registration of 3D Points to 3D Lines with Application to the Pose Estimation in a Projective Image," IBM RC 20560, 1996.

[8] S. Schreiner, et al, "A system for percutaneous delivery of treatment with a fluoroscopically-guided robot," presented at Joint Conf. of Computer Vision, Virtual Reality, and Robotics in Medicine and Medical Robotics and Computer Surgery, Grenoble, France, 1997.

[9] J. M. Boone, J. A. Seibert, et al, "Analysis and correction of imperfections in the image intensified-TV-digitizer imaging chain," Medical Physics, vol. 18, pp. 236-242, 1991.

[10] F. L. Bookstein, Morphometric tools for landmark data, Geometry and biology: Cambridge University Press, 1991.

[11] G. Wolberg, Digital Image Warping: IEEE Computer Society Press Monograph, 1990.

[12] G. Levy, "Robotic Control for Digital Subtraction Radiography," in Electrical Engineering. Rutgers, the State University of New York, 1994, pp. 145. 